\title{
Female pediatric and adolescent genitalia trauma: a retrospective analysis of the National Trauma Data Bank
}

\author{
Shannon M. Fan ${ }^{1} \cdot$ Areg Grigorian ${ }^{1} \cdot$ Haris H. Chaudhry ${ }^{1}$ Angela Allen ${ }^{1} \cdot$ Beatrice Sun ${ }^{1} \cdot$ Nathan Jasperse ${ }^{1}$. \\ Spencer Albertson ${ }^{1} \cdot$ Jeffry Nahmias ${ }^{1}$
}

Accepted: 20 August 2020 / Published online: 26 August 2020

(c) Springer-Verlag GmbH Germany, part of Springer Nature 2020

\begin{abstract}
Purpose Pediatric genitalia injury represents $0.6 \%$ of all pediatric trauma. It is crucial for providers to understand whether pediatric patients are at risk for violent mechanisms, such as rape, assault, or other abuse. Therefore, we sought to perform a large database analysis of pediatric and adolescent female genitalia trauma, comparing mechanisms of injury (i.e., sexual abuse) and need for operative intervention between adolescent and pediatric cohorts.

Methods The National Trauma Data Bank was queried (years 2007-2015) for female patients $\leq 16$ years old with external genitalia (vaginal or vulvar) trauma. Two groups were compared: pediatrics $(<12$ years old) and adolescents $(12-16$ years old).

Results Out of 303,992 female patients, 3206 (1.1\%) were identified to have genitalia trauma with the majority being pediatric patients $(92.1 \%)$ and with injury to the vagina $(62.6 \%)$. Pediatric patients with vaginal injury were less likely to be victims of rape $(4.1 \%$ vs. $17.3 \%, p<0.001)$ and assault $(2.1 \%$ vs. $7.2 \%, p<0.001)$ but more likely to be victims of other abuse $(9.5 \%$ vs. $3.4 \%, p=0.003)$. More of the adolescent patients with vaginal trauma required repair $(58.7 \%$ vs. $43.2 \%, p<0.001)$. Pediatric patients with injury to the vulva were less likely to be victims of rape $(0.7 \%$ vs. $2.8 \%, p=0.01)$ and motor vehicle accidents $(4.2 \%$ vs. $11.0 \%, p<0.001)$.

Conclusion Genitalia trauma occurs in $1.1 \%$ of pediatric and adolescent trauma cases with the vagina being more commonly injured compared to the vulva. Adolescent patients with vaginal injuries were more likely to be victims of rape and assault and required repair more often, while those with vulvar injuries were more likely due to motor vehicle accidents. Health care providers must be aware of these at-risk populations and the differences between them to identify female victims of violence and provide resources to assist with recovery.
\end{abstract}

Keywords Pediatric trauma $\cdot$ Genitalia injury $\cdot$ Sexual abuse $\cdot$ Vaginal trauma $\cdot$ Vulva trauma

\section{Introduction}

Trauma is one of the leading causes of pediatric mortality in the United States (US) with an additional 9.2 million children presenting annually to US emergency departments

Electronic supplementary material The online version of this article (https://doi.org/10.1007/s00383-020-04736-7) contains supplementary material, which is available to authorized users.

Jeffry Nahmias

jnahmias@hs.uci.edu

1 Division of Trauma, Burns and Surgical Critical Care, Department of Surgery, University of California, Irvine, 333 The City Blvd West, Suite 1600, Orange, CA 92868-3298, USA for non-fatal injuries [1]. However, pediatric genitalia injuries comprise a small fraction of this population, with an incidence ranging $0.2-8 \%$ of all reported pediatric trauma cases [2-5]. These pediatric genitalia injuries can occur due to accidental trauma (i.e. straddle injuries, motor vehicle accidents, etc.) or due to abuse and as a result can lead to long-term physical and/or psychological sequelae for the victims $[5,6]$.

A multi-center study between 1993 and 1997 found that perineal injuries requiring surgery in female pediatric patients were most commonly associated with motor vehicle accidents, however they did not specifically evaluate for sexual assault [2]. Another multi-institutional study between 2003 and 2007 found that sexual assault was the third most common cause of pediatric female genitalia trauma with an 
incidence of $11.5 \%$ [7]. Several single-center studies have identified straddle injuries as the most common cause of unintentional female pediatric genitalia trauma, whereas hymenal injury is suggestive of sexual abuse [4, 8]. Furthermore, populations within pediatric patients, such as adolescents (12-16 years old) and younger pediatric patients $(<12$ years old), may receive different examination methods, as well as have different mechanisms of injury, injury patterns, and outcomes [9-12]. Therefore, we sought to perform a large national analysis using a pediatric trauma database to describe the demographics, injury patterns, treatments, and outcomes associated with pediatric and adolescent genitalia trauma (i.e., vaginal and vulvar injuries). In addition, we hypothesized a difference in mechanisms of injury (i.e., sexual abuse) and need for operative intervention between adolescent and pediatric cohorts.

\section{Methods}

This study protocol was approved by our local Institutional Review Board. We performed a retrospective analysis using the National Trauma Data Bank (NTDB) of female trauma patients $\leq 16$ years old with vaginal or vulva trauma from January 2007 through December 2015. The NTDB is known to be the largest trauma registry with a dataset including over 1.2 million cases of patients aged $<20$ years old aggregated from over 700 national trauma centers from 2007 to 2015 [13]. Vaginal trauma was defined using the International Classification of Diseases Version-9 (ICD-9) diagnosis codes: 878.6, 878.7. Vulvar trauma was defined using the ICD-9 diagnosis codes: $878.4,878.5$. Two groups were compared: pediatric patients $<12$ years old and adolescent patients between 12 and 16 years old. The primary outcome was operative intervention for vaginal/vulvar trauma. ICD-9 procedure codes were used to identify patients undergoing operative intervention and are listed in Appendix-A.

Frequency statistics were performed for all variables. The differences in demographic characteristics, mechanism of injury, and injury-associated characteristics including injury severity score (ISS) were collected. Demographic information included age, race, and alcohol/drug use. The injury profile included ISS and severe grade (grade $>3$ ) on the abbreviated injury scale (AIS) for the head, thorax and abdomen. Other measured outcomes included hospital length of stay (LOS), ventilator days, urinary tract infection, and mortality. A Student's $t$ test and Mann-Whitney $U$ test were used to compare continuous variables and reported with medians and interquartile range or as means with standard deviation. Chi-square test was used to compare categorical variables and reported as percentages.

All $p$ values were two-sided, with a statistical significance level of $<0.05$. All missing data points were not imputed but treated as missing data. All analyses were performed with IBM SPSS Statistics for Windows (Version 24, IBM Corp., Armonk, NY).

\section{Results}

\section{Characteristics of all pediatric and adolescent injuries}

From the 303,992 female pediatric trauma patients $\leq 16$ years old between years 2007 and 2015, 3,206 (1.1\%) were identified to have genitalia trauma. The most common mechanisms of injury to genitalia were being pedestrian struck (45.6\%) and blunt injury not including pedestrian struck, falls, and motor vehicle and bicycle accidents (15.4\%), followed by abuse not including rape (6.8\%), falls (6.7\%), bicycle accidents (5.5\%), motor vehicle accidents (5.1\%), stab wounds (4.2\%), and rape (3.6\%). Of the 3206 patients, 2953 $(92.1 \%)$ were pediatric patients, and 2007 (62.6\%) had injury to the vagina. The most common mechanisms of vaginal injury were being pedestrian struck (46.8\%) and blunt injury not including pedestrian struck, falls, and motor vehicle and bicycle accidents $(11.7 \%)$, followed by abuse not including rape $(8.9 \%)$, falls $(5.9 \%)$, rape $(5.4 \%)$, motor vehicle accidents (5.2\%), bicycle accidents (4.7\%), and stab wounds (4.2\%). Vulvar injuries were most commonly caused by being pedestrian struck (43.9\%), blunt injury not including pedestrian struck, falls, and motor vehicle and bicycle accidents $(20.9 \%)$, followed by falls $(8.0 \%)$, bicycle accidents (6.7\%), motor vehicle accidents (4.9\%), stab wounds (4.2\%), and abuse not including rape (3.6\%). A total of $44.8 \%$ of vaginal injuries and $54.6 \%$ of vulvar injuries underwent operative repair.

\section{Comparison of pediatric vs. adolescent vaginal injuries}

The median ages for patients with injury to the vagina were 6 years for pediatric patients and 14 years for adolescents $(p<0.001)$. When compared to the adolescent group, pediatric patients with injury to the vagina were more likely to be of white race $(61.2 \%$ vs. $50.0 \%, p=0.002)$ and less likely to be of black race $(16.4 \%$ vs. $27.8 \%, p<0.001)$ and screen positive for drugs of abuse $(0.4 \%$ vs. $3.8 \%, p<0.001)$. Pediatric patients had a lower median ISS (1.0 vs. 2.0, $p<0.001)$ and lower severe grade for the AIS of the thorax $(0.3 \%$ vs. $1.5 \%$, $p=0.01$ ). Pediatric patients were less likely to be victims of rape $(4.1 \%$ vs. $17.3 \%, p<0.001)$, assault $(2.1 \%$ vs. $7.2 \%$, $p<0.001)$, gunshot wounds $(0.3 \%$ vs. $2.4 \%, p<0.001)$, and motor vehicle accidents ( 4.3 vs. $13.5 \%, p<0.001$ ). However, they were more likely to be victims of other abuse $(9.5 \%$ vs. $3.4 \%, p=0.003)$ and blunt injury not including pedestrian 
struck, falls, and motor and bicycle accidents (12.4\% vs. $5.7 \%, p=0.01$ ) when compared to adolescents (Table 1).

Pediatric patients with vaginal trauma had a shorter median LOS ( 1 vs. 2 days, $p<0.001$ ) and were less likely to have vaginal trauma repairs $(43.2 \%$ vs. $58.7 \%, p<0.001)$, compared to adolescent patients. There was no difference in intensive care unit (ICU) days ( 3 vs. 3 days, $p=0.77$ ), ventilator days ( 2 vs. 3 days, $p=0.15$ ), and mortality rate ( $1.8 \%$ vs. $1.0 \%, p=0.40$ ) between the two cohorts (Table 2).

\section{Comparison of pediatric vs. adolescent vulvar injuries}

Of the female patients $\leq 16$ years old, 1349 (42.1\%) patients had vulvar trauma, of which 1204 (89.3\%) were pediatric patients and $145(10.7 \%)$ were adolescents. Pediatric patients and adolescents with injury to the vulva had median ages of 6 and 13, respectively ( $p<0.001)$. Compared to adolescents, pediatric patients had a lower mean ISS (3.2 vs. 4.6, $p<0.001)$ and were less likely to be have a positive toxicology screen $(0.5 \%$ vs. $4.1 \%, p=0.02)$. Pediatric patients were less likely to be victims of rape $(0.7 \%$ vs. $2.8 \%, p=0.01)$, gunshot wounds $(0.2 \%$ vs. $1.4 \%, p=0.01)$, and motor vehicle accidents ( $4.2 \%$ vs. $11.0 \%, p<0.001)$, but more likely to be victims of blunt injury not including pedestrian struck, falls, and motor and bicycle accidents (22.5\% vs. 7.5\%) (Table 3).

Pediatric patients also had a shorter median LOS (1 vs. 2 days, $p<0.001)$. However, there was no difference in rates of operative repair to the vulva for pediatric patients as compared to adolescent patients $(54.6 \%$ vs. $54.5 \%, p=0.98)$. Additionally, there was no difference in ICU days (2 vs. 3 days, $p=0.09$ ), ventilator days ( 2 vs. $2.5, p=0.97$ ), and mortality rate $(0.9 \%$ vs. $0.0 \%, p=0.26)$ between pediatric and adolescent patients with vulvar injuries (Table 4).
Table 1 Patient characteristics of pediatric and adolescent external genitalia trauma to the vagina

\begin{tabular}{|c|c|c|c|}
\hline \multirow[t]{2}{*}{ Characteristic } & \multicolumn{2}{|l|}{ Vagina } & \multirow[t]{2}{*}{$p$ value } \\
\hline & Pediatric $(n=1813)$ & Adolescents $(n=208)$ & \\
\hline Age, years, median (IQR) & $6.0(4)$ & $14.0(2)$ & $<0.001$ \\
\hline ISS, median (IQR) & $1.0(3)$ & $2.0(8)$ & $<0.001$ \\
\hline \multicolumn{4}{|l|}{ Race, $n(\%)$} \\
\hline White & $1109(61.2 \%)$ & $104(50.0 \%)$ & 0.002 \\
\hline Black & $298(16.4 \%)$ & $58(27.8 \%)$ & $<0.001$ \\
\hline Asian & $33(1.8 \%)$ & $3(1.4 \%)$ & 0.06 \\
\hline Hispanic ethnicity, $n(\%)$ & $253(14.0 \%)$ & $24(11.6 \%)$ & 0.53 \\
\hline Alcohol, $n(\%)$ & $9(0.4 \%)$ & $5(2.4 \%)$ & 0.24 \\
\hline Drug, $n(\%)$ & $7(0.4 \%)$ & $8(3.8 \%)$ & $<0.001$ \\
\hline \multicolumn{4}{|l|}{ Mechanism, $n(\%)$} \\
\hline Rape & $74(4.1 \%)$ & $36(17.3 \%)$ & $<0.001$ \\
\hline Other abuse & $173(9.5 \%)$ & $7(3.4 \%)$ & 0.003 \\
\hline Other assault & $38(2.1 \%)$ & $15(7.2 \%)$ & $<0.001$ \\
\hline \multicolumn{4}{|l|}{ Penetrating } \\
\hline Gunshot wound & $5(0.3 \%)$ & $5(2.4 \%)$ & $<0.001$ \\
\hline Stab wound & $78(4.3 \%)$ & $7(3.4 \%)$ & 0.52 \\
\hline Other & $18(1.0 \%)$ & $1(0.5 \%)$ & 0.47 \\
\hline \multicolumn{4}{|l|}{ Blunt } \\
\hline Pedestrian struck & $849(46.8 \%)$ & $96(46.2 \%)$ & 0.39 \\
\hline Falls & $105(5.8 \%)$ & $14(6.7 \%)$ & 0.09 \\
\hline Motor vehicle accident & $78(4.3 \%)$ & $28(13.5 \%)$ & $<0.001$ \\
\hline Bicycle accident & $89(4.9 \%)$ & $6(2.9 \%)$ & 0.19 \\
\hline Other & $225(12.4 \%)$ & $12(5.7 \%)$ & 0.01 \\
\hline All other & $366(20.2 \%)$ & $83(39.9 \%)$ & \\
\hline \multicolumn{4}{|l|}{ Severe $^{\mathrm{a}}$ AIS, $n(\%)$} \\
\hline Head & $38(2.1 \%)$ & $5(2.4 \%)$ & 0.77 \\
\hline Spine & $1(0.1 \%)$ & $0(0.0 \%)$ & 0.73 \\
\hline Thorax & $5(0.3 \%)$ & $3(1.4 \%)$ & 0.01 \\
\hline Abdomen & $19(1.0 \%)$ & $4(1.9 \%)$ & 0.26 \\
\hline
\end{tabular}

$I Q R$ interquartile range, ISS injury severity score, $A I S$ abbreviated injury score

${ }^{\text {a }}$ Severe (grade $>3$ ) 
Table 2 Patient outcomes of pediatric and adolescent external genitalia trauma to the vagina
Table 3 Patient characteristics of pediatric and adolescent external genitalia trauma to the vulva

\begin{tabular}{lllc}
\hline Characteristic & \multicolumn{2}{l}{ Vagina } & $p$ value \\
\cline { 2 - 3 } & Pediatric $(n=1414)$ & Adolescents $(n=208)$ & \\
\hline Outcomes & & & $<0.001$ \\
LOS, days, median (IQR) & $1.0(1)$ & $2.0(4)$ & 0.77 \\
ICU, days, median (IQR) & $3.0(4)$ & $3.0(4)$ & 0.15 \\
Ventilator, days, median (IQR) & $2.0(4)$ & $3.0(4)$ & 0.33 \\
Urinary tract infection, $n(\%)$ & $3(0.2 \%)$ & $1(0.5 \%)$ & $<0.001$ \\
Procedure, $n(\%)$ & & $122(58.7 \%)$ & \\
Repair of vagina & $784(43.2 \%)$ & - & 0.40 \\
$\quad$ Repair of vulva or perineum & - & $2(1.0 \%)$ & \\
Mortality, $n(\%)$ & $31(1.8 \%)$ & &
\end{tabular}

$I Q R$ interquartile range, $L O S$ length of stay, $I C U$ intensive care unit

\begin{tabular}{|c|c|c|c|}
\hline \multirow[t]{2}{*}{ Characteristic } & \multicolumn{2}{|l|}{ Vulva } & \multirow[t]{2}{*}{$p$ value } \\
\hline & Pediatric $(n=1204)$ & Adolescents $(n=145)$ & \\
\hline Age, years, median (IQR) & $6.0(4)$ & $13.0(2)$ & $<0.001$ \\
\hline ISS, mean (SD) & $3.2(6)$ & $4.6(7)$ & $<0.001$ \\
\hline \multicolumn{4}{|l|}{ Race, $n(\%)$} \\
\hline White & $710(59.0 \%)$ & $89(61.4 \%)$ & 0.58 \\
\hline Black & $188(15.6 \%)$ & $23(15.9 \%)$ & 0.92 \\
\hline Asian & $29(2.4 \%)$ & $2(1.3 \%)$ & 0.41 \\
\hline Hispanic ethnicity, $n(\%)$ & $178(14.8 \%)$ & $20(13.8 \%)$ & 0.53 \\
\hline Alcohol, $n(\%)$ & $8(0.6 \%)$ & $5(3.4 \%)$ & 0.24 \\
\hline Drug, $n(\%)$ & $6(0.5 \%)$ & $6(4.1 \%)$ & 0.02 \\
\hline \multicolumn{4}{|l|}{ Mechanism, $n(\%)$} \\
\hline Rape & $8(0.7 \%)$ & $4(2.8 \%)$ & 0.01 \\
\hline Other abuse & $47(3.9 \%)$ & $1(0.7 \%)$ & 0.48 \\
\hline Other assault & $19(1.6 \%)$ & $1(0.7 \%)$ & 0.40 \\
\hline \multicolumn{4}{|l|}{ Penetrating } \\
\hline Gunshot wound & $2(0.2 \%)$ & $2(1.4 \%)$ & 0.01 \\
\hline Stab wound & $50(4.2 \%)$ & $6(4.1 \%)$ & 0.99 \\
\hline Other & $12(1.0 \%)$ & $1(0.7 \%)$ & 0.72 \\
\hline \multicolumn{4}{|l|}{ Blunt } \\
\hline Pedestrian stuck & $524(43.5 \%)$ & $68(46.9 \%)$ & 0.29 \\
\hline Falls & $98(8.1 \%)$ & $10(6.9 \%)$ & 0.14 \\
\hline Motor vehicle accident & $50(4.2 \%)$ & $16(11.0 \%)$ & $<0.001$ \\
\hline Bicycle accident & $75(6.2 \%)$ & $15(10.3 \%)$ & 0.06 \\
\hline Other & $271(22.5 \%)$ & $11(7.5 \%)$ & 0.01 \\
\hline All other & $122(10.1 \%)$ & $16(11.0 \%)$ & \\
\hline \multicolumn{4}{|l|}{ Severe $^{\mathrm{a}}$ AIS, $n(\%)$} \\
\hline Head & $22(1.8 \%)$ & $1(0.7 \%)$ & 0.31 \\
\hline Spine & $1(0.1 \%)$ & $0(0.0 \%)$ & 0.73 \\
\hline Thorax & $4(0.3 \%)$ & $0(0.0 \%)$ & 0.48 \\
\hline Abdomen & $6(0.5 \%)$ & $3(2.1 \%)$ & 0.28 \\
\hline
\end{tabular}

$I Q R$ interquartile range, ISS injury severity score, $A I S$ abbreviated injury score

${ }^{\text {a }}$ Severe $($ grade $>3)$ 
Table 4 Patient outcomes of pediatric and adolescent external genitalia trauma to the vulva

\begin{tabular}{lllc}
\hline Characteristic & \multicolumn{2}{l}{ Vulva } & $p$ value \\
\cline { 2 - 3 } & Pediatric $(n=2023)$ & Adolescents $(n=145)$ & \\
\hline Outcomes & & & $<0.001$ \\
LOS, days, median (IQR) & $1.0(1)$ & $1.5(2)$ & 0.09 \\
ICU, days, median (IQR) & $2.0(3)$ & $3.0(4)$ & 0.97 \\
Ventilator, days, median (IQR) & $2.0(3)$ & $2.5(4)$ & 0.73 \\
Urinary tract infection, $n(\%)$ & $1(0.1 \%)$ & $1(0.7 \%)$ & \\
Procedure, $n(\%)$ & & & 0.98 \\
Repair of vagina & - & $79(54.5 \%)$ & 0.26 \\
$\quad$ Repair of vulva or perineum & $657(54.6 \%)$ & $0(0.0 \%)$ & \\
Mortality, $n(\%)$ & $10(0.9 \%)$ & &
\end{tabular}

$I Q R$ interquartile range, $L O S$ length of stay, $I C U$ intensive care unit

\section{Discussion}

This retrospective study comprising eight years of national data from the NTDB found that just over $1 \%$ of female patients $\leq 16$ years old had genitalia trauma. The vagina was more commonly injured than the vulva in both pediatric and adolescent patients. However, adolescent patients were more likely to be victims of rape, had longer LOS than pediatric patients, and were more likely to undergo repair of vaginal injuries. Additionally, vulvar injuries in adolescent patients were more likely to be secondary to motor vehicle accidents.

Pediatric genitalia trauma is scarcely studied, potentially due to its taboo nature. In a retrospective study of 41 children's hospitals, Shnorhavorian et al. found that the mechanism of pediatric genitalia injuries among 5664 patients was most often due to unintentional injury with an object (15.8\%), followed by home accidents $(14.3 \%)$ and sexual assaults (11.5\%) [7]. However, our findings demonstrate that although the most common mechanisms are blunt injury from being pedestrian struck (45.6\%) and blunt injury not including pedestrian struck, falls, and motor vehicle and bicycle accidents (15.4\%), the next most common mechanism is intentional abuse, excluding rape (6.8\%). This suggests that abuse should be considered whenever female patients $\leq 16$ years old present with external genitalia injury. In addition, trauma providers should evaluate female children involved in significant blunt trauma mechanisms for these injuries.

Previous studies, albeit mixed with perineal and anorectal injuries, have found that surgical repair is uncommon and only performed in up to $9 \%$ of female genitalia injuries $[2,7,14]$. However, in a retrospective review, Spitzer et al. found that around $21 \%$ of unintentional pediatric vulvar trauma required surgical repair [4]. In contrast, our study found nearly $50 \%$ of all female pediatric patients with external genitalia trauma underwent operative repair. However, given that our database is focused on trauma patients, lesser injured pediatric patients are not captured within this database, thus skewing our data. That said, trauma providers unfamiliar with these injuries should be cognizant of this common requirement for operative intervention and liberally seek specialist consultation from gynecologists and/ or abuse specialist to ensure proper operative treatment is offered when appropriate.

Vaginal injures may be indicative of pediatric non-accidental trauma. Joki-Erkkila et al. found that the median age of children with police-reported suspicion of sexual abuse between 2001 and 2009 was 7.1 years of age [15]. A case series by Berkoff et al. sought to evaluate whether genitalia examination was effective in determining sexual abuse, and found that vaginal discharge along with posterior hymenal transections, deep notches, and perforations could indicate sexual abuse in prepubescent female [16]. We add to this literature by further dividing injuries based on anatomy (vaginal vs. vulvar) and age group (pediatrics vs. adolescent patients), demonstrating that common mechanisms of injury change based on vaginal or vulvar injury and pediatric or adolescent population. Our study was consistent in that we found a greater rate of vaginal injuries was caused by rape or assault, with $\sim 17 \%$ of vaginal injuries in adolescent patients due to rape. Moreover, Spitzer et al. found that patients who required surgical repair had a greater association with certain risk factors, such as older age and injuries involving penetration and the hymen [4]. In support of this, our study demonstrated a high surgical repair rate in patients with vaginal injuries with a significantly higher repair rate in adolescents.

Straddle injury, including the vulva, as compared to hymenal injury is more likely to occur in accidental genitalia trauma, compared to sexual assault. Spitzer et al. found that straddle injuries, which do not involve the vagina, were the most common cause of unintentional female pediatric trauma, making up $81.9 \%$ of injuries [4]. Bond et al. similarly found that unintentional injuries were more commonly associated with straddle injury, involving the labia minora in $67.9 \%$ of the pediatric patients between ages 1 and 12 
[8]. Similarly, our study found a higher rate of vulvar injuries was due to blunt injury, as compared to rape or assault (85.0\% vs. $5.9 \%)$. In contrast, we found a significantly greater cause of injury by motor vehicle accidents in adolescents as compared to pediatrics. Therefore, adolescents in motor vehicle accidents should raise heightened concern for vulvar injury. These findings further suggest that a detailed history and physical examination is prudent to ensure identification of external genitalia trauma after blunt accidental mechanisms of injury, such as motor vehicle accidents.

\section{Limitations}

Our study has several limitations including reporting bias, misclassifications, and missing data, which are inherent to retrospective database studies. In addition, we were limited to identifying genitalia trauma to only vaginal and vulvar injuries, lacking more granular information, such as time from the reported mechanism of injury, type of abuse or assault, and more details regarding the severity of the injuries (e.g., American Association for the Surgery of Trauma Grade of Injury) and involvement of other perineal structures and/or hymen. Moreover, data regarding the exact operative intervention and the type of provider performing these interventions were also unavailable. In addition, as mentioned, the NTDB only includes patients seen at a verified trauma center by the trauma team. Thus, patients with relatively minor traumatic injuries seen solely by an emergency room physician were not accounted for in this study, thereby likely overestimating the number of injuries that require repair. Additionally, given that the trauma team sees a limited portion of this population, these patients are likely biased towards those with more severe injuries. Finally, as a retrospective database study, with the database confined to the index hospitalization, we are unable to determine causeand-effect associations and lack follow-up on our patient population, including outcomes related to operative repairs and psychological outcomes. Future prospective multicenter studies with long-term follow-up should be performed to evaluate these important outcomes. However, despite these limitations, our study is strengthened by the large nationwide population.

\section{Conclusion}

In a large national database study of female pediatric patients $\leq 16$ years old with genitalia trauma limited to the vaginal and vulvar areas, there was a greater rate of vaginal injuries compared to vulvar injuries. Adolescent patients were more likely to be victims of rape and assault when compared to younger pediatric patients. Both adolescents and younger pediatric patients required a high rate of operative intervention for vaginal injuries, with adolescents more commonly requiring operative repair. These findings emphasize the value and need for provider vigilance when caring for pediatric patients involved in these mechanisms and/or use of this information to help identify pediatric patients sustaining external genitalia injuries from both accidental and non-accidental trauma.

Funding No funding was received for this work. This research did not receive any specific grant from funding agencies in the public, commercial, or not-for-profit sectors.

\section{Compliance with ethical standards}

Conflict of interest The authors declare that they have no conflicts of interest.

Ethical approval This research involved humans. However, since this retrospective study was performed using a national database with deidentified patients, risk to participants is minimal.

Informed consent There is no consent required.

\section{References}

1. Avraham JB, Bhandari M, Frangos SG, Levine DA, Tunik MG, DiMaggio CJ (2019) Epidemiology of paediatric trauma presenting to US emergency departments: 2006-2012. Inj Prev 25(2):136-143. https://doi.org/10.1136/injuryprev-2017-042435

2. Scheidler MG, Schultz BL, Schall L, Ford HR (2000) Mechanisms of blunt perineal injury in female pediatric patients. J Pediatr Surg 35(9):1317-1319. https://doi.org/10.1053/ jpsu.2000.9315

3. Tarman GJ, Kaplan GW, Lerman SL, McAleer IM, Losasso BE (2002) Lower genitourinary injury and pelvic fractures in pediatric patients. Urology 59(1):123-126. https://doi.org/10.1016/ s0090-4295(01)01526-6 (discussion 126)

4. Spitzer RF, Kives S, Caccia N, Ornstein M, Goia C, Allen LM (2008) Retrospective review of unintentional female genital trauma at a pediatric referral center. Pediatr Emerg Care 24(12):831-835. https://doi.org/10.1097/PEC.0b013e31818ea064

5. Casey JT, Bjurlin MA, Cheng EY (2013) Pediatric genital injury: an analysis of the National Electronic Injury Surveillance System. Urology 82(5):1125-1130. https://doi. org/10.1016/j.urology.2013.05.042

6. Pokorny SF (1997) Genital trauma. Clin Obstet Gynecol 40(1):219-225. https://doi.org/10.1097/00003081-19970 3000-00023

7. Shnorhavorian M, Hidalgo-Tamola J, Koyle MA, Wessells H, Larison C, Goldin A (2012) Unintentional and sexual abuserelated pediatric female genital trauma: a multiinstitutional study of free-standing pediatric hospitals in the United States. Urology 80(2):417-422. https://doi.org/10.1016/j.urology.2012.04.021

8. Bond GR, Dowd MD, Landsman I, Rimsza M (1995) Unintentional perineal injury in prepubescent girls: a multicenter, prospective report of 56 girls. Pediatrics 95(5):628-631 
9. Jacobs AM, Alderman EM (2014) Gynecologic examination of the prepubertal girl. Pediatr Rev 35(3):97-104. https://doi. org/10.1542/pir.35-3-97

10. Yilmaz AE, Celik N, Soylu G, Donmez A, Yuksel C (2012) Comparison of clinical and microbiological features of vulvovaginitis in prepubertal and pubertal girls. J Formos Med Assoc 111(7):392-396. https://doi.org/10.1016/j.jfma.2011.05.013

11. Price J (2013) Injuries in prepubertal and pubertal girls. Best Pract Res Clin Obstet Gynaecol 27(1):131-139. https://doi. org/10.1016/j.bpobgyn.2012.08.008

12. Boyle C, McCann J, Miyamoto S, Rogers K (2008) Comparison of examination methods used in the evaluation of prepubertal and pubertal female genitalia: a descriptive study. Child Abuse Negl 32(2):229-243. https://doi.org/10.1016/j.chiabu.2007.06.004

13. ACoSCo T (2016) National Trauma Data Bank 2016: Pediatric Annual Report. Chicago, IL
14. Jones JG, Worthington $\mathrm{T}$ (2008) Genital and anal injuries requiring surgical repair in females less than 21 years of age. J Pediatr Adolesc Gynecol 21(4):207-211. https://doi.org/10.1016/j. jpag.2007.10.010

15. Joki-Erkkila M, Niemi J, Ellonen N (2018) Child sexual abuseinitial suspicion and legal outcome. Forensic Sci Int 291:39-43. https://doi.org/10.1016/j.forsciint.2018.06.032

16. Berkoff MC, Zolotor AJ, Makoroff KL, Thackeray JD, Shapiro RA, Runyan DK (2008) Has this prepubertal girl been sexually abused? JAMA 300(23):2779-2792. https://doi.org/10.1001/ jama.2008.827

Publisher's Note Springer Nature remains neutral with regard to jurisdictional claims in published maps and institutional affiliations. 